\title{
Efficient Pipeline Processing of Crowdsourcing Workflows
}

\author{
Ken Mizusawa \\ University of Tsukuba \\ ken.mizusawa.2016b@mlab.info
}

\author{
Keishi Tajima \\ Kyoto University \\ tajima@i.kyoto-u.ac.jp
}

\author{
Masaki Matsubara \\ University of Tsukuba \\ masaki@slis.tsukuba.ac.jp
}

\author{
Toshiyuki Amagasa \\ University of Tsukuba \\ amagasa@cs.tsukuba.ac.jp
}

\author{
Atsuyuki Morishima \\ University of Tsukuba \\ mori@slis.tsukuba.ac.jp
}

\begin{abstract}
This paper addresses the pipeline processing of sequential workflows in crowdsourcing. Sequential workflows consisting of several subtasks are ubiquitous in crowdsourcing. Our approach is to control the budget distribution to subtasks in order to balance the execution speed of the subtasks and to improve throughput of overall sequential workflows. As we cannot control the price for earlier steps retrospectively in the stepwise batch execution, we explore pipeline processing schemes. Our experimental results show that our pipeline processing scheme with price control achieves significantly higher throughput of sequential workflows.
\end{abstract}

\section{CCS CONCEPTS}

- Information systems $\rightarrow$ Crowdsourcing;

\section{KEYWORDS}

Crowdsourcing; price control; improved throughput

\section{ACM Reference format:}

Ken Mizusawa, Keishi Tajima, Masaki Matsubara, Toshiyuki Amagasa, and Atsuyuki Morishima. 2018. Efficient Pipeline Processing of Crowdsourcing Workflows. In Proceedings of The 27th ACM International Conference on Information and Knowledge Management, Torino, Italy, October 22-26, 2018 (CIKM '18), 4 pages.

https://doi.org/10.1145/3269206.3269292

\section{INTRODUCTION}

Today, the problems handled by crowdsourcing are becoming more complex, and we need to use workflows that involve more than one type of subtasks and dataflow among them. Figure 1 shows a well-known example [3] of a sequential workflow for revising documents with three types of subtasks: "Find," "Fix," and "Verify." Each Find task asks workers to point out the location in a sentence to be revised; the Fix task asks workers to revise the phrases at these locations; and the Verify task asks workers to decide whether the revision is appropriate.

Permission to make digital or hard copies of all or part of this work for personal or classroom use is granted without fee provided that copies are not made or distributed for profit or commercial advantage and that copies bear this notice and the full citation on the first page. Copyrights for components of this work owned by others than the author(s) must be honored. Abstracting with credit is permitted. To copy otherwise, or republish, to post on servers or to redistribute to lists, requires prior specific permission and/or a fee. Request permissions from permissions@acm.org.

CIKM '18, October 22-26, 2018, Torino, Italy

() 2018 Copyright held by the owner/author(s). Publication rights licensed to Association for Computing Machinery.

ACM ISBN 978-1-4503-6014-2/18/10 . \$ \$15.00

https://doi.org/10.1145/3269206.3269292

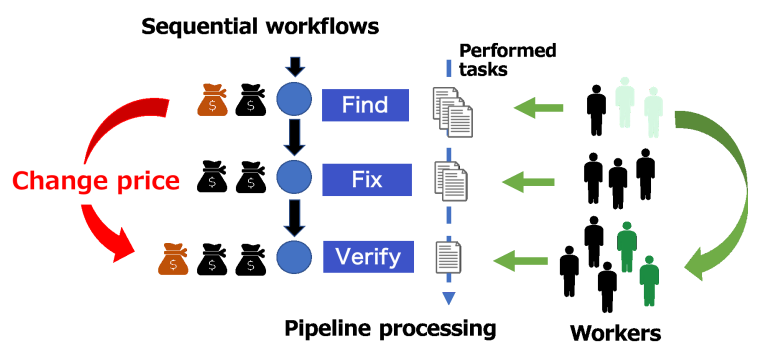

Figure 1: Pipeline processing with price control
We can use this workflow to crowdsource a task of revising a document as follows. First, we divide the document into a set of sentences. Second, we generate a set of Find tasks for these sentences. We then obtain the task results, generate the Fix tasks, obtain the task results, and generate the Verify tasks. Recently, a common practice in crowdsourcing is to submit tasks to crowdsourcing services in a stepwise batch style. That is, we submit all the subsequent tasks (e.g., Fix) after all the current tasks (e.g., Find) are completed.

However, this stepwise style has a disadvantage. In crowdsourcing, it is difficult to predict the crowd's behavior and we often encounter cases where the task completion rate is less (or more) than expected. One approach to this problem is to reserve some budget and use it when we find some slow tasks. That is, when we find some type of tasks are slow, we increase their price in order to accelerate them. In the stepwise style, however, it is difficult to optimize the budget distribution over the entire workflow because we cannot change the price for the earlier subtasks after we know the behavior of the later subtasks.

In this paper, we explore pipeline processing of such workflows instead of the batch style. In a pipeline execution, subsequent tasks are generated immediately after some of the preceding tasks are completed. In addition to the trivial advantage that some of the final results are produced earlier, pipeline processing allows us to dynamically control the budget distribution over the entire workflow. For example, we can initially allocate the same amount of budget to all the three types of tasks explained above, and if some of them are slower than expected while some of them are fast enough, we can change the budget distribution among the three tasks, expecting that workers will change their choice of tasks. Unlike pure machine processing, stepwise processing and pipeline processing of the same workflow do not show the same total processing time because tasks are performed by humans whose execution speed and choice of tasks are affected by various factors such as price settings. 
The contributions of this paper are as follows.

Pipeline processing of workflows with price control. This paper proposes a pipeline processing framework for sequential workflows with a dynamic price control scheme. The framework gives us a principled way to change payments for tasks according to the progress of different tasks.

Experiments with real-world workers. This paper presents the results of our extensive experiments comparing the proposed scheme with a non-pipelined, stepwise batch processing scheme. In the experiments, we examined the results of 14 executions on different days and at different times for each scheme. Our experimental results show that the completion time of the proposed pipeline processing framework with payment control was 1.6 to 1.8 times faster than that of the stepwise batch processing scheme, without affecting the data quality. Our statistical analysis showed that there were differences in performance at the $5 \%$ significance level.

The remainder of this paper is organized as follows. Section 2 explains the related work. Section 3 describes the proposed pipeline processing framework with dynamic budget control. Section 4 reports our experimental results. Section 5 concludes the paper and presents future work.

\section{RELATED WORK}

Workflows combining microtasks with dataflow among them have been recognized as a promising approach to solving a variety of problems [1][4][9][11][13].

One of the interesting problems pertains to finding a good workflow among a variety of those that achieve the same goal. There are researches on SQL-based systems [14], and others that focus on particular workflow patterns to select good workflows based on the patterns [5][8]. In addition, research on how to exploit crowd power to find such workflows has been reported [12]. However, these studies focused on how to construct the workflow, rather than adjusting the prices for tasks during their execution.

Pricing tasks is an important issue in crowdsourcing. Several studies have been reported on how tasks should be priced within the budget considering the latency and completion of the tasks, assuming that the workers follow specific worker models [6][7][10]. Some studies considered both the price and the number of duplicate tasks required to satisfy the required quality of results [2]. These studies assumed a set of the same tasks that are independent of each other (i.e., there is no dataflow among them). However, we can borrow the idea of introducing worker models to better predict worker behavior, which is an interesting prospect for future work.

The problem setting in [15] is similar to ours in that it deals with the pricing problem in complex workflows. However, their aim was to improve data quality rather than efficiency. Their proposal controls the distribution of budgets in the horizontal direction, where the problem is how to price the whole task path for each data item to improve the data quality on average. By contrast, our proposal controls the distribution of budgets in the vertical direction, where the problem is how to price each task on the path through which data proceed, with the ultimate aim of improving throughput. In future research, it would be interesting to combine these two techniques, which are complementary to each other, in

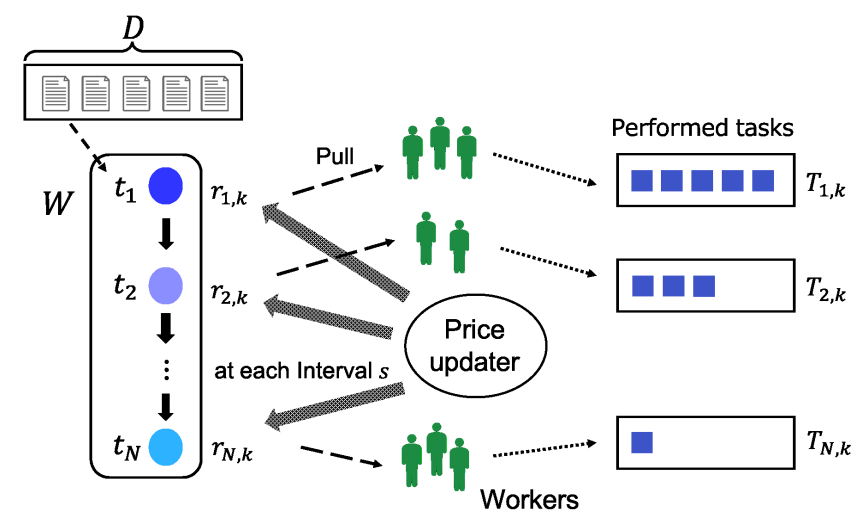

Figure 2: Payment change based on the number of uncompleted tasks

an effort to explore a larger search space to find good pricing from different perspectives.

\section{PROPOSED FRAMEWORK}

This section explains the proposed framework for dynamically changing the price setting according to the progress of the workflow.

\subsection{Overview}

Figure 2 provides an overview of the framework. The inputs are as follows: (1) a workflow (sequence of tasks) $W=$ $\left[t_{1}, t_{2}, \ldots, t_{i}, \ldots, t_{N}\right]$, where each time a worker performs a task instance of $t_{i}$, the result is used to generate a task instance of $t_{i+1} ;(2)$ the number $D$ of data items that will go through the workflow; (3) the total budget $B$; and (4) the interval $s$ for updating the task price. In addition, the framework requires (5) $p$, a parameter defining the degree of price change, and optionally includes (6) the minimum and maximum price for a task.

Given the inputs, the framework generates tasks for $t_{i}$ and periodically updates the prices for the tasks according to the progress of execution. The framework monitors the progress status of the tasks at each interval $s$, and computes the price $r_{i, k}$ for each $t_{i}$ for the $k$-th interval according to their status at that time.

\subsection{Initial Setting}

Let $r_{i, 0}$ be the initial price for performing $t_{i}$. The initial price will be used from the beginning to the time before the first interval $s$ has passed. When the interval is reached, we compute the price for the next period, denoted by $r_{i, 1}$. We repeat this at each interval.

Initially (for the time period between 0 to $s$ ), we assign the same amount of budget $B / D$ to each of the $D$ data items, and distribute them equally to the $N$ tasks in $W$ :

$$
r_{i, 0}=\frac{B}{D \cdot N} \quad \text { for } 1 \leq i \leq N
$$




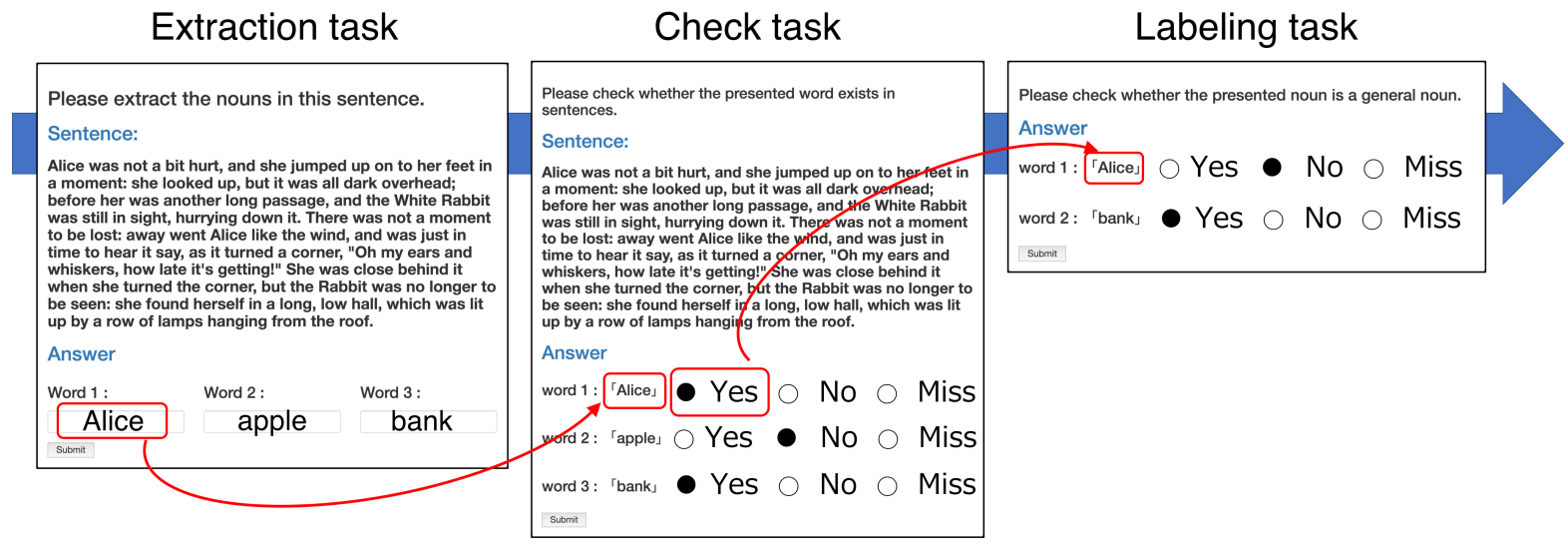

Figure 3: Task overviews of sequential workflow in the experiment

\subsection{Updating Prices}

At each interval, the framework computes the price for the next period. Let $r_{i, k}$ be the price. Let $T_{i, k}$ be the number of task instances of $t_{i}$ that workers have already completed at time $k \cdot s$. Then, we compute the weight $r_{i, k}^{\prime}$ to compute $r_{i, k}$ as follows:

$$
r_{i, k}^{\prime}=\left(\frac{D-T_{i, k}}{\sum_{j=1}^{N}\left(D-T_{j, k}\right)}\right)^{p} .
$$

This means that we assign larger weights to tasks with more task instances that have not yet been performed. The parameter $p$ controls the degree of the influence over $r_{i, k}^{\prime}$.

Then, we normalize $r_{i, k}^{\prime}$ so that $\sum_{i=1}^{N} r_{i, k}^{\prime}=1$, and compute $r_{i, k}$ as follows.

$$
r_{i, k}=N \cdot r_{i, 0} \cdot r_{i, k}^{\prime}
$$

where the sum of $r_{i, k}$ s does not exceed $B / D$.

For example, suppose that $r_{i, 0}=0.04$ and $D=50$; further, $T_{i, k}$ is 30,20, 10 for $t_{1}, t_{2}$, and $t_{3}$, respectively. If $p=1$, then $r_{1, k}, r_{2, k}$, and $r_{3, k}$ are $0.0267,0.0400$, and 0.0533 , respectively. If $p=3$, these become $0.0096,0.0327$, and 0.0775 , respectively.

In special cases, such as just after the workflow has started, we do not have task instances for some tasks in the workflow. In such cases, we reserve $B /(D \cdot N)$ budget for the future execution of each of such tasks.

The minimum and maximum price can be specified if necessary. For example, Amazon Mechanical Turk has a minimum price (\$0.01). When we need to round values, we usually conduct a truncation such that the total value does not exceed the budget. The only exception to this is the minimum value constraint. If $r_{i, k}$ is less than the minimum value, we round the value up to the minimum value. This can lead to budget overflow. In such a case, we need to adjust the prices of other tasks to guarantee that the tasks are completed within the budget.

\section{EXPERIMENT}

We conducted an experiment by comparing four methods (one stepwise batch execution and three pipeline processing) in a sequential workflow. For the three pipeline processing methods, we used a different parameter $p$, which represents the degree of price changes, as described in Section 3: "Pipeline_p0" did not change prices $(p=0)$, "Pipeline_p1" changed prices slightly $(p=1)$, and "Pipeline_p3" changed prices considerably $(p=3)$. We ran the workflow 14 times with each scheme.

\subsection{Settings}

Workflow: We used a sequential workflow to label nouns that appear in documents (Figure 3). The workflow consisted of three tasks. First, the Extraction task asked workers to extract three nouns from sentences and enter them in a textbox. Second, the Check task asked workers to check whether the nouns extracted by another worker certainly existed in the sentences. Finally, the Labeling task asked workers to label the nouns that survived the Check task with "Yes" when they were common nouns.

Data: We extracted 14 passages (with approximately 120 words in each passage) from three novels: "Alice's Adventures in Wonderland," "The Adventures of Sherlock Holmes," and "Peter Pan," which we retrieved from Project Gutenberg ${ }^{1}$. In the experiment, we focused on the task processing time excluding the time spent by the server, such as the time for generating and issuing tasks. To exclude it as much as possible, we pre-computed the data shown in each task, but each task was actually issued only after the corresponding previous task was completed by some worker.

Procedure: We conducted 14 sets of comparisons among the four methods. The number of data items that proceeded through the workflow was $50(D=50)$, such that the total number of tasks was 8,400 ( 50 data $\times 3$ tasks $\times 4$ methods $\times 14$ sets). All tasks were submitted via Amazon Mechanical Turk. Workers could perform only one task instance for each task. Owing to the Amazon Mechanical Turk fee setting, a fraction less than 0.01 was truncated. The dates of 14 submissions of every method span all days of the week and

\footnotetext{
${ }^{1}$ http://www.gutenberg.org
} 
all time in the day in a balanced way to remove the influences of the time of the submission.

Parameters: The interval $s$ was set to 2 min for updating prices in pipeline processing. Similarly, for the stepwise approach (hereafter "Stepwise"), we monitored the progress every 2 min to determine when to submit the tasks for the next step. The total budget $B$ was set to $\$ 6.00$. As a result, the initial price $r_{i, 0}$ was $\$ 0.04$ ( $\$ 6.00 / 50$ data / 3 tasks).

\subsection{Results}

Figure 4 shows a distribution of the total processing time of 14 runs of each method. Multiple comparison tests with Bonferroni correction revealed significant differences between Stepwise and Pipeline_p1 $(\mathrm{p}<.01)$, and between Stepwise and Pipeline_p3 $(\mathrm{p}<.05)$ in the total processing time. By contrast, there was no significant difference between Stepwise and Pipeline_p0 $(p=0.358)$, which did not change the prices.

For Pipeline_p1 and Pipeline_p3, both of which change prices, the average total payments after 14 executions were $\$ 5.51$ and $\$ 5.62$, and the maximum payments were $\$ 5.70$ and $\$ 5.85$, respectively; they never exceeded $\$ 6.00$. The standard errors were $\$ 0.0274$ and $\$ 0.0399$, respectively. The prices were changed 18 times with Pipeline_p1 and 35 times with Pipeline_p3. The difference in the accuracy of the results of the four methods was not statistically significant $(81 \%, 82 \%, 86 \%$, and $82 \%$ for Stepwise, Pipeline_p0, Pipeline_p1, and Pipeline_p3, respectively).

\subsection{Discussion}

Our experiments suggest that a relatively simple framework for changing prices can achieve a significant improvement in the completion time. However, our careful observation of the log suggested that there are cases where we need to be more careful, and where there is room to revise the framework.

First, we noticed that our simple framework always set cheaper prices for tasks that appeared earlier in the workflow. This risks stagnating the workflow, if all workers wait for the more lucrative tasks in the later stages-although it should be noted that we did not observe any such stagnation in our experiments.

Second, we noticed that the current framework has no means to stimulate the execution when there are no workers to perform tasks at current prices. As the price is set according to the number of the tasks performed already, the framework cannot change the price in such a situation.

Thus, we believe that we can further improve the performance of the pipeline framework in several ways, and we intend to explore this in our future work.

\section{CONCLUSION}

We proposed an efficient method for pipeline processing of sequential workflows that involve microtasks. The pipeline framework allows us to design principled ways to dynamically change the price of tasks. We conducted an extensive comparison of the proposed methods with the standard stepwise batch processing scheme. The results showed that pipeline processing with dynamic pricing was up to 1.8 times faster on average over 14 runs than stepwise batch execution with fixed prices.

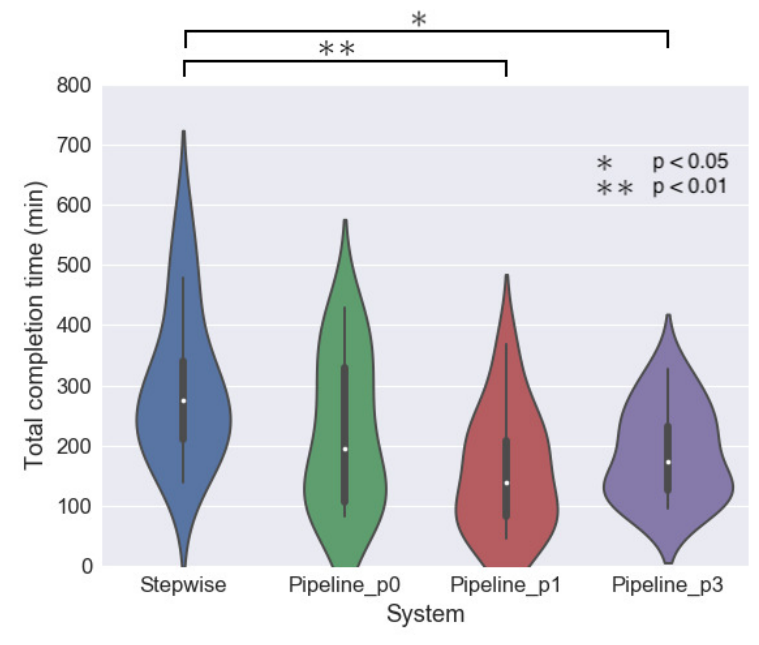

Figure 4: Violin plot of total completion time of each method

In future work, we plan to extend our framework to a variety of workflows other than sequential ones, and we will explore more sophisticated strategies for price changes.

\section{ACKNOWLEDGMENTS}

This work was supported by JST CREST Grant Number JPMJCR16E3, Japan.

\section{REFERENCES}

[1] V. Ambati, S. Vogel, and J. Carbonell. 2012. Collaborative workflow for crowdsourcing translation. In Proc. of ACM CSCW'12. ACM, 1191-1194.

[2] D. W Barowy, C. Curtsinger, E. D Berger, and A. McGregor. 2012. Automan: A platform for integrating human-based and digital computation. Acm Sigplan Notices 47, 10 (2012), 639-654.

[3] M. S Bernstein, G. Little, R. C Miller, B. Hartmann, M. S Ackerman, D. R Karger, D. Crowell, and K. Panovich. 2015. Soylent: a word processor with a crowd inside. Commun. ACM 58, 8 (2015), 85-94.

[4] L. B Chilton, G. Little, D. Edge, D. S Weld, and J. Landay. 2013. Cascade: Crowdsourcing taxonomy creation. In Proc. of SIGCHI'13. ACM, 1999-2008.

[5] P. Dai, C. H Lin, D. S Weld, et al. 2013. Pomdp-based control of workflows for crowdsourcing. Artificial Intelligence 202 (2013), 52-85.

[6] S. Faradani, B. Hartmann, and P. G Ipeirotis. 2011. What's the Right Price? Pricing Tasks for Finishing on Time. Human computation 11 (2011), 11.

[7] Y. Gao and A. Parameswaran. 2014. Finish them!: Pricing algorithms for human computation. PVLDB'14 7, 14 (2014), 1965-1976.

[8] S. Goto, T. Ishida, and D. Lin. 2016. Understanding Crowdsourcing Workflow: Modeling and Optimizing Iterative and Parallel Processes. In Proc. HCOMP 2016.

[9] D. Haas, J. Ansel, L. Gu, and A. Marcus. 2015. Argonaut: macrotask crowdsourcing for complex data processing. PVLDB'15 8, 12 (2015), 1642-1653.

[10] Z. Hu and J. Zhang. 2017. Optimal posted-price mechanism in microtask crowdsourcing. In Proc. of IfCAI'17. AAAI Press, 228-234.

[11] A. Kittur, B. Smus, S. Khamkar, and R. E Kraut. 2011. Crowdforge: Crowdsourcing complex work. In Proc. of UIST'11. ACM, 43-52.

[12] A. Kulkarni, M. Can, and B. Hartmann. 2012. Collaboratively crowdsourcing workflows with turkomatic. In Proc. of ACM CSCW'12. ACM, 1003-1012.

[13] C. Lofi and K. El Maarry. 2014. Design patterns for hybrid algorithmiccrowdsourcing workflows. In Business Informatics (CBI), 2014 IEEE 16th Conference on, Vol. 1. IEEE, 1-8.

[14] A. G. Parameswaran, H. Park, H. Garcia-Molina, N. Polyzotis, and J. Widom. 2012. Deco: declarative crowdsourcing. In Proc. of ACM CIKM'12. ACM, 1203-1212.

[15] L. Tran-Thanh, T. D. Huynh, A. Rosenfeld, S. D Ramchurn, and N. R Jennings. 2015. Crowdsourcing Complex Workflows under Budget Constraints. In AAAI. 1298-1304. 